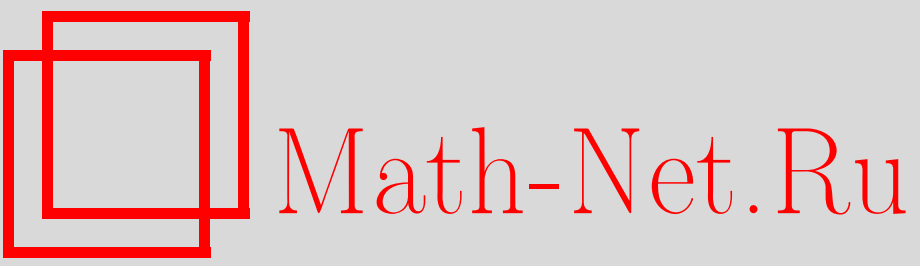

А. Ю. Колесов, Н. Х. Розов, О природе явления буферности в слабо диссипативных системах, ТMФ, 2006, том 146, номер 3, 447-466

DOI: https://doi.org/10.4213/tmf2047

Использование Общероссийского математического портала Math-Net.Ru подразумевает, что вы прочитали и согласны с пользовательским соглашением

http://www.mathnet.ru/rus/agreement

Параметры загрузки:

IP: 54.210 .77 .194

26 апреля 2023 г., 04:08:23 
ТЕОРЕТИЧЕСКАЯ

И МАТЕМАТИЧЕСКАЯ

ФИЗИКА

Том 146, № 3

март, 2006

(C) 2006 г.

А. Ю. Колесов ${ }^{*}$ Н. Х. Розов

\title{
О ПРИРОДЕ ЯВЛЕНИЯ БУФЕРНОСТИ В СЛАБО ДИССИПАТИВНЫХ СИСТЕМАХ
}

\begin{abstract}
Предлагается механизм накапливания аттракторов в слабо диссипативных конечномерных системах, суть которого заключается в следующем. Пусть некоторая гамильтонова или консервативная система с полутора или более степенями свободы возмущена малыми добавками, обеспечивающими ее диссипативность. При определенных условиях и при стремлении возмущений к нулю количество ее аттракторов, возникающих в малых окрестностях различных эллиптических состояний равновесия или циклов невозмущенной системы, может неограниченно возрастать. Рассмотрены содержательные примеры из механики и радиофизики: модели динамики подскакивающего мяча, ускорения Ферми, линейного осциллятора с ударами и автогенератора с дискретной последовательностью $R L C$-контуров в цепи обратной связи.
\end{abstract}

Ключевые слова: аттракторы, периодические движения, явление буферности, отображения, релейные системы.

\section{1. ВВЕДЕНИЕ}

О феномене буферности принято говорить, если в фазовом пространстве некоторой динамической системы при подходящем выборе параметров можно гарантировать сосуществование любого фиксированного числа однотипных аттракторов (состояний равновесия, циклов, торов и т.д.).

Из результатов известной статьи Витта [1], а также из значительно более поздних работ [2], [3] следует, что буферность характерна для широкого класса физических процессов, описывающихся гиперболическими уравнениями (например, для автоколебательных процессов в распределенных электрических и механических системах). При этом ситуация, наиболее благоприятная для реализуемости указанного явления, заключается в следующем. Предположим, что в задаче об устойчивости нулевого положения равновесия некоторой гиперболической системы дифференци-

* Ярославский государственный университет, Ярославль, Россия.

E-mail: kolesov@uniyar.ac.ru

${ }^{\dagger}$ Московский государственный университет, Москва, Россия.

E-mail:rozov@rozov.mccme.ru 
альных уравнений имеет место критический случай счетного числа чисто мнимых собственных значений, а при изменении каких-либо входящих в эту систему параметров происходит смещение части спектра устойчивости в правую комплексную полуплоскость. Тогда, как установлено в уже упоминавшихся работах [1]-[3], при отсутствии определенных резонансных соотношений между собственными частотами системы чаще всего в ней наблюдается феномен буферности в простейшем его варианте. А именно, сосуществуют несколько квазигармонических (т.е. близких к гармоническим по времени) устойчивых циклов, число которых за счет подходящего выбора параметров может быть сделано сколь угодно большим.

Описанный механизм накапливания устойчивых стационарных режимов характерен, естественно, только для систем с бесконечномерным фазовым пространством. Однако, как показано ниже, буферность может возникать и в конечномерных системах при условии, что они близки к консервативным.

Действительно, рассмотрим некоторую гамильтонову, или консервативную, систему обыкновенных дифференциальных уравнений (в физике такие системы часто называют обратимыми, так как они не меняются при обращении времени) с полутора или более степенями свободы. Тогда, как отмечено, например, в книгах [4], [5], в общем случае в такой системе хаотические движения сосуществуют со счетным числом так называемых островков устойчивости, примыкающих к эллиптическим состояниям равновесия или циклам. Это означает, что внешние хаотические траектории не могут попасть внутрь упомянутых островков, и наоборот, любая траектория из островка, которая в простейшем случае является периодической или квазипериодической, остается в нем при всех $t \in \mathbb{R}$ (в более сложных случаях внутри островка, в свою очередь, имеются изолированные хаотические движения, перемежающиеся другими островками, и т.д.).

Предположим теперь, что наша система возмущена малыми добавками, обеспечивающими ее диссипативность. Тогда некоторые из эллиптических состояний равновесия или циклов, о которых говорилось выше, могут стать асимптотически устойчивыми. Таким образом, на месте островков устойчивости возникают аттракторы и, что самое главное, количество последних может неограниченно увеличиваться при стремлении возмущений к нулю. А это, напомним, и означает, что в рассматриваемой системе наблюдается явление буферности.

Следует отметить, что в случае обыкновенных дифференциальных уравнений существуют и другие, значительно более сложные механизмы накапливания устойчивых циклов, обусловленные наличием в системах так называемых гомоклинических касаний (см., например, [6]-[9]). Среди большого количества результатов, полученных для такого рода систем, остановимся лишь на трех, имеющих непосредственное отношение к нашей тематике. В связи с этим сначала рассмотрим $C^{r}$-гладкую $(r \geqslant 4)$ систему обыкновенных дифференциальных уравнений в $\mathbb{R}^{3}$ и предположим, что у нее существует изолированное состояние равновесия $O$ с характеристическими корнями $\lambda_{1,2}=-\gamma \pm i \omega(\gamma>0, \omega \neq 0), \quad \lambda_{3}>0$. Предположим, что имеется гомоклиническая к $O$ траектория $Г$. Тогда при $\sigma_{1}=\operatorname{Re} \lambda_{1}+\lambda_{3}>0, \sigma_{2}=2 \operatorname{Re} \lambda_{1}+\lambda_{3}<0$ в классе таких систем плотны системы со счетным множеством устойчивых периодических движений [7]. 
Второй результат, аналогичный описанному выше, принадлежит Ньюхаусу [9]. Пусть $p$ - гиперболическая седловая неподвижная точка $C^{r}$-диффеоморфизма $f$ в $\mathbb{R}^{2}$, для которого $\operatorname{det} f^{\prime}(p)<1$, а устойчивое и неустойчивое многообразия точки $p$ касаются в некоторой точке $p_{0}$. Тогда в сколь угодно малой $C^{r}$-окрестности $f$ существует диффеоморфизм $\tilde{f}$, имеющий бесконечно много устойчивых периодических орбит.

Третий результат, принадлежащий Гаврилову и Шильникову [6], заключается в том, что появлению или исчезновению точки гомоклинического касания предшествуют каскады бифуркаций типа седло-узел. При этих бифуркациях рождаются пары, состоящие из устойчивого и неустойчивого циклов, причем количество устойчивых периодических движений за счет подходящего выбора бифуркационных параметров может быть сделано сколь угодно большим. Конкретные примеры, в которых реализуется указанный сценарий возникновения буферности, хорошо известны. Это уравнение Дуффинга с малой диссипацией и малым периодическим внешним воздействием, а также уравнение колебаний маятника с малым затуханием и вибрирующей точкой подвеса (см. [10] и приведенную там библиографию).

В настоящей работе мы не рассматриваем приложений, в которых возникновение буферности связано с гомоклиническими бифуркациями, так как наша цель - дать представление о феномене буферности как можно более широкому кругу читателей, далеких от указанной тематики. В связи с этим при подборе материала мы сознательно остановили свой выбор на ряде содержательных примеров из механики и радиофизики, анализ которых не требует громоздких математических выкладок. Желающих ознакомиться с предметом более подробно отсылаем к книгам [11].

\section{2. ЯВЛЕНИЕ БУФЕРНОСТИ В СИСТЕМАХ С МЕХАНИЧЕСКИМИ СОУДАРЕНИЯМИ}

Простейшей системой из интересующего нас класса является модель повторных соударений шара с массивным столом, колеблющимся по синусоидальному закону. Эта модель представляет собой дискретное отображение следующего вида [10]:

$$
\begin{aligned}
& v_{n+1}=\alpha v_{n}-\gamma \cos \left(\varphi_{n}+v_{n}\right), \\
& \varphi_{n+1}=\varphi_{n}+v_{n} \quad(\bmod 2 \pi),
\end{aligned}
$$

где $v_{n}, \quad n=0,1, \ldots,-$ нормированная скорость отскакивающего мяча после $n$-го соударения со столом, $\varphi_{n+1}-\varphi_{n}-$ время его полета между соседними соударениями (определенное по модулю $2 \pi$ ), параметр $\alpha \in(0,1)$ характеризует диссипацию, $\gamma>0$ - амплитуда колебаний стола.

Покажем, что явление буферности в отображении (1) наблюдается в случае слабой диссипации, а именно, при

$$
\alpha=1-\varepsilon, \quad 0<\varepsilon \ll 1 .
$$

Для того чтобы убедиться в этом, перейдем при условии (2) к каноническим переменным $I_{n}=v_{n}, \theta_{n}=\varphi_{n}+v_{n}-\pi / 2$. В результате интересующее нас отображение примет вид

$$
I_{n+1}=(1-\varepsilon) I_{n}+K \sin \theta_{n}, \quad \theta_{n+1}=\theta_{n}+I_{n+1} \quad(\bmod 2 \pi),
$$

4 Теоретическая и математическая физика, т. 146, № 3, 2006 г. 
где $K=\gamma$. Нетрудно заметить, что отображение (3) имеет серию неподвижных точек

$$
O_{m}(\varepsilon)=\left(\theta_{(m)}, I_{(m)}\right), \quad m=0, \pm 1, \pm 2, \ldots,
$$

где $I_{(m)}=2 \pi m$, номер $m$ удовлетворяет оценке

$$
2 \pi|m| \varepsilon \leqslant K
$$

a $\theta_{(m)}-$ один из корней уравнения

$$
K \sin \theta=z, \quad z=2 \pi m \varepsilon .
$$

Ясно также, что при $\varepsilon \rightarrow 0$ количество неподвижных точек неограниченно растет. Что же касается устойчивости указанных неподвижных точек, то она определяется по расположению корней уравнений

$$
\lambda^{2}-\left(2+K \cos \theta_{(m)}-\varepsilon\right) \lambda+1-\varepsilon=0 .
$$

Последующий анализ разобьем на два этапа. Пусть сначала параметр $K$ в $(3)$ фиксирован и удовлетворяет неравенству

$$
K<4
$$

Обозначим через $\theta=\theta_{0}(z) \quad(|z|<K)$ корень уравнения (6), принадлежащий интервалу $(\pi / 2,3 \pi / 2)$. Справедливо следующее утверждение.

ТеОРема 1. Пусть выполнено условие (8). Тогда по любому фиксированному $z_{*} \in(0, K)$ можно указать такое достаточно малое $\varepsilon_{0}=\varepsilon_{0}\left(z_{*}\right)>0$, что при всех $0<\varepsilon \leqslant \varepsilon_{0}$ отображение (3) имеет экспоненциально устойчивые неподвижные точки (4), где

$$
\theta_{(m)}=\left.\theta_{0}(z)\right|_{z=2 \pi \varepsilon m}, \quad 2 \pi \varepsilon|m| \leqslant z_{*}
$$

ДокАЗАТЕЛЬСтво. Обратимся к уравнению (7) и заметим, что при условии

$$
\left|2+K \cos \theta_{(m)}-\varepsilon\right|<2 \sqrt{1-\varepsilon}
$$

его корни $\lambda_{m}^{1}, \lambda_{m}^{2}$ комплексные и $\left|\lambda_{m}^{1}\right|=\left|\lambda_{m}^{2}\right|=\sqrt{1-\varepsilon}<1$. Если верны соотношения (9), требование (10) заведомо выполняется при всех достаточно малых $\varepsilon$, так как при $|z| \leqslant z_{*}$

$$
\left|2+K \cos \theta_{0}(z)\right|<2 \text {. }
$$

Действительно, эта оценка эквивалентна условию

$$
\sqrt{K^{2}-z^{2}}<4
$$

справедливость которого при всех $|z| \leqslant z_{*}$ есть следствие неравенства (8). Теорема доказана. 
Заметим, что согласно доказанной теореме количество устойчивых неподвижных точек отображения (3) при $\varepsilon \rightarrow 0$ неограниченно растет (имеет порядок $\varepsilon^{-1}$ ), а значит, при условии (8) в рассматриваемой системе реализуется феномен буферности. Механизм возникновения этого явления в данном случае именно тот, о котором говорилось во введении. Для пояснения ситуации положим сначала в $(3) \varepsilon=0$. Тогда, как нетрудно заметить, получившееся отображение имеет счетное число неподвижных точек

$$
(\theta, I)=(\pi, 2 \pi m), \quad m \in \mathbb{Z},
$$

каждая из которых при условии (8) является эллиптической (см. рис. 1, на котором изображен фрагмент $\{(\theta, I): 0 \leqslant \theta \leqslant 2 \pi,|I| \leqslant 10\}$ фазового портрета отображения (3) при $K=2, \quad \varepsilon=0)$. При переходе от $\varepsilon=0$ к малым $\varepsilon>0$ неподвижные точки (13) становятся устойчивыми фокусами, причем "выживает" асимптотически большое, но конечное их число.

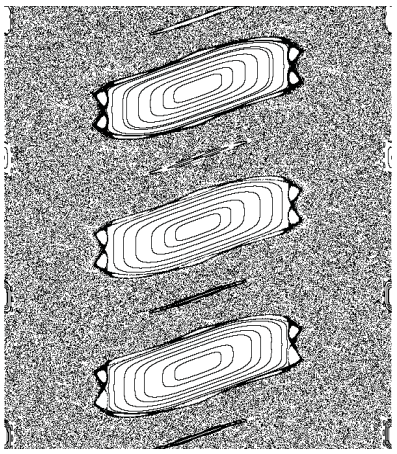

Рис. 1

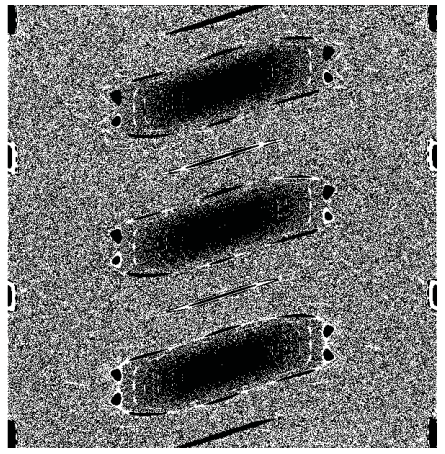

Рис. 2

Обнаруженный механизм возникновения буферности иллюстрирует рис. 2, где показано изменение фазового портрета отображения (3) в случае $K=2$ при переходе от $\varepsilon=0$ к значению $\varepsilon=0.0001$. Три больших темных пятна на этом рисунке как раз и соответствуют трем состояниям равновесия (13) (при $m=0, \pm 1$ ), ставшим устойчивыми. Добавим еще, что помимо неподвижных точек отображение (3) имеет при $\varepsilon>0$ устойчивые циклы различных периодов (на рис. 2 некоторым из них соответствуют темные пятна меньших размеров). Однако области их притяжения существенно у́же, чем у неподвижных точек.

Возвращаясь к исходной системе (1), заметим, что физический смысл имеют только неподвижные точки (4) с неотрицательными номерами $m$. Буферность в данной системе означает, что в зависимости от выбора начальной скорости шара и начальной фазы колебаний стола реализуется тот или иной периодический режим из имеющегося достаточно большого потенциального их запаса.

Предположим теперь, что справедливо неравенство

$$
K>4 \text {. }
$$

Тогда условие устойчивости (12) заведомо нарушается при малых $|z|$, т.е. неустойчивыми становятся все неподвижные точки (4), (9) с номерами $m \sim 1$. Выполняться 
неравенство (12) будет лишь при $|z|>\sqrt{K^{2}-16}$. Тем самым, приходим к следующему утверждению.

Теорема 2. При условии (14) по любым фиксированным $z_{*}, z_{* *}$ таким, что

$$
\sqrt{K^{2}-16}<z_{*}<z_{* *}<K,
$$

можно указать такое $\varepsilon_{0}=\varepsilon_{0}\left(z_{*}, z_{* *}\right)>0$, что при всех $0<\varepsilon \leqslant \varepsilon_{0}$ у отображения (3) экспоненциально устойчивыми являются неподвижные точки (4), компоненты $\theta_{(m)}$ которых задаются соотношениями

$$
\theta_{(m)}=\left.\theta_{0}(z)\right|_{z=2 \pi \varepsilon m}, \quad z_{*} \leqslant 2 \pi \varepsilon|m| \leqslant z_{* *} .
$$

Из этой теоремы следует, что при выполнении условия (14) буферность сохраняется, так как количество неподвижных точек, определяемых формулами (4), (15), по-прежнему асимптотически велико (имеет порядок $\varepsilon^{-1}$ ). Однако появляется и новый аспект теории: устойчивыми могут быть только неподвижные точки с асимптотически большими значениями $|m|$.

Заканчивая обсуждение динамики системы (3), сделаем одно замечание общего характера. Для этого обозначим через П отображение, получающееся из отображения (3) при $\varepsilon=0$, и предположим, что данное отображение имеет цикл периода $s \geqslant 2$ с начальным условием $P_{0}=\left(\theta_{0}, I_{0}\right)$, т.е. $\Pi^{s}\left(P_{0}\right)=P_{0}$. Предположим далее, что матрица линеаризации

$$
A_{s}=\left(\Pi^{s}\right)^{\prime}\left(P_{0}\right)
$$

(правая часть данного равенства есть значение производной отображения $\Pi^{s}$ по текущей строке, выполненное в точке $P_{0}$ ) на указанном цикле удовлетворяет неравенству

$$
\left|\operatorname{tr} A_{s}\right|<2 .
$$

Тогда справедливо следующее утверждение.

Teорема 3. Найдется такое достаточно малое $\varepsilon_{0}>0$, что при всех $0<\varepsilon \leqslant \varepsilon_{0}$ отображение (3) имеет экспоненциально устойчивый цикл периода s с аналитически зависящим от в начальным условием $P(\varepsilon), P(0)=P_{0}$.

ДокАЗАТЕЛьСтво. Существование цикла очевидно. Действительно, в силу предполагаемой грубости цикла при $\varepsilon=0$ (см. (16), (17)) он сохраняется при всех достаточно малых $\varepsilon>0$ и, более того, его начальное условие $P(\varepsilon), P(0)=P_{0}$, аналитически зависит от $\varepsilon$. Рассмотрим соответствующую матрицу линеаризации $A_{s}(\varepsilon), A_{s}(0)=A_{s}$. Снова привлекая неравенство (17), убеждаемся, что собственные значения $\lambda_{1}(\varepsilon), \lambda_{2}(\varepsilon)$ этой матрицы комплексные, $\left|\lambda_{1}(\varepsilon)\right|=\left|\lambda_{2}(\varepsilon)\right|=(1-\varepsilon)^{s / 2}<1$. Последнее является следствием того, что якобиан отображения (3) во всех точках равен $1-\varepsilon$. Теорема доказана. 
Необходимо добавить, что область применимости теоремы 3 заведомо не пуста. Для того чтобы убедиться в этом, отметим, что при $\varepsilon=0$ у отображения (3) существует, например, счетное число циклов периода 2 с начальными условиями

$$
\left(\theta_{0}, I_{0}\right)=(\pi,(2 m+1) \pi), \quad m \in \mathbb{Z}
$$

Каждый из этих циклов является эллиптическим при $K<2$ и седловым при $K>2$. Это означает, что при любом фиксированном $K<2$ и при всех достаточно малых $\varepsilon>0$ циклы периода 2 с начальными условиями, близкими к $(18)$, становятся устойчивыми, причем, как и в случае состояний равновесия (4), выживает асимптотически большое (порядка $\left.\varepsilon^{-1}\right)$, но конечное их число.

Еще один пример системы с механическими соударениями в сущности представляет собой некоторую модификацию предыдущего. Рассмотрим частицу, движущуюся между неподвижной и осциллирующей стенками (модель ускорения Ферми). Эволюция такой системы описывается отображением Улама (см., например, [12])

$$
\begin{aligned}
u_{n+1} & =\left|(1-\varepsilon) u_{n}+\sin \varphi_{n}\right|, \\
\varphi_{n+1} & =\varphi_{n}+\frac{2 \pi M}{u_{n+1}} \quad(\bmod 2 \pi) .
\end{aligned}
$$

Здесь $u_{n}$ - приведенная скорость частицы, $0<\varepsilon \ll 1$ - относительная потеря скорости при столкновении с неподвижной стенкой, $\varphi_{n}-$ фаза колеблющейся стенки в момент соударения с частицей, а параметр $M \gg 1$ пропорционален отношению расстояния между стенками к амплитуде колебаний стенки.

Покажем, что при подходящем выборе параметров в системе (19) реализуется явление буферности. С этой целью сделаем в ней замену $I_{n}=\varepsilon u_{n}$ и будем предполагать, что

$$
\frac{1}{M}=\varepsilon \beta, \quad \beta=\text { const }>1 .
$$

Отсюда получим более удобное для последующего анализа отображение

$$
\begin{aligned}
I_{n+1} & =\left|(1-\varepsilon) I_{n}+\varepsilon \sin \varphi_{n}\right|, \\
\varphi_{n+1} & =\varphi_{n}+\frac{2 \pi}{\beta I_{n+1}} \quad(\bmod 2 \pi) .
\end{aligned}
$$

Действительно, исследование неподвижных точек этого отображения не вызывает затруднений: таковыми являются точки

$$
(\varphi, I)=\left(\varphi_{(m)}, I_{(m)}\right), \quad m=1,2, \ldots,
$$

где $I_{(m)}=1 /(\beta m)$, а $\varphi_{(m)}-$ корень уравнения

$$
\sin \varphi=\frac{1}{\beta m}
$$

из интервала $(0, \pi / 2)$ (другой корень этого уравнения брать не имеет смысла, так как ему отвечает заведомо неустойчивая неподвижная точка). Подчеркнем, что в силу условия $\beta>1$ уравнение (23) разрешимо при любом натуральном $m$. 
За устойчивость неподвижных точек (22) отвечает расположение корней $\lambda_{m}^{1}, \lambda_{m}^{2}$ уравнения

$$
\lambda^{2}-\left(2-\varepsilon-2 \pi \varepsilon m \sqrt{\beta^{2} m^{2}-1}\right) \lambda+1-\varepsilon=0,
$$

анализ которого также не вызывает затруднений. В самом деле, при любом фиксированном $m$ и при всех достаточно малых $\varepsilon>0$ в силу очевидного неравенства

$$
\left|2-\varepsilon-2 \pi \varepsilon m \sqrt{\beta^{2} m^{2}-1}\right|<2 \sqrt{1-\varepsilon}
$$

имеем $\left|\lambda_{m}^{1}\right|=\left|\lambda_{m}^{2}\right|=\sqrt{1-\varepsilon}$. Это означает, что, как и в предыдущем случае, в рамках отображения (21) мы сталкиваемся с феноменом буферности: устойчивыми являются его неподвижные точки $(22)$ с номерами $m=1, \ldots, m_{*}(\varepsilon)$, где $m_{*}(\varepsilon) \rightarrow \infty$ при $\varepsilon \rightarrow 0$. Добавим еще, что номер $m_{*}(\varepsilon)$ здесь обязательно конечен, так как при любом фиксированном $\varepsilon>0$ и при $m \rightarrow \infty$ у уравнения $(24)$ появляется корень, модуль которого больше единицы.

Анализ систем с механическими соударениями завершим рассмотрением примера, в котором фазовое пространство уже не является циклическим по одной из переменных. А именно, предположим, что имеется линейный осциллятор с трением $\varepsilon$, $0<\varepsilon \ll 1$, и собственной частотой $\omega_{0}>0$, испытывающий удары в моменты времени $t=n T$, где $T>0, n \in \mathbb{Z}$. Тогда, следуя [5], в качестве уравнения движения такого осциллятора можно взять

$$
\ddot{x}+\varepsilon \dot{x}+\omega_{0}^{2} x=-\frac{\omega_{0} K}{T} \sin x \sum_{n=-\infty}^{\infty} \delta\left(\frac{t}{T}-n\right),
$$

где через $\delta(t)$, как обычно, обозначена $\delta$-функция, а $K>0$ - безразмерный параметр, пропорциональный амплитуде возмущения.

Из (25) непосредственным интегрированием выводим соотношения

$$
x\left(t_{n}+0\right)=x\left(t_{n}-0\right), \quad \dot{x}\left(t_{n}+0\right)=\dot{x}\left(t_{n}-0\right)-K \omega_{0} \sin x\left(t_{n}-0\right), \quad n \in \mathbb{Z},
$$

где $t_{n}=n T$. В случае $t \neq t_{n}$ решение уравнения (25) удовлетворяет уравнению свободного движения

$$
\ddot{x}+\varepsilon \dot{x}+\omega_{0}^{2} x=0,
$$

которое позволяет выразить значения $x_{n+1}=x\left(t_{n+1}-0\right), \dot{x}_{n+1}=\dot{x}\left(t_{n+1}-0\right)$ через $x_{n}=x\left(t_{n}-0\right), \dot{x}_{n}=\dot{x}\left(t_{n}-0\right)$. Используя (26), на этом пути получаем отображение

$$
\begin{aligned}
& u_{n+1}=(1-\varepsilon \alpha)\left[\left(u_{n}+K \sin v_{n}\right) \cos \alpha+v_{n} \sin \alpha\right]-\varepsilon\left(u_{n}+K \sin v_{n}\right) \sin \alpha, \\
& v_{n+1}=(1-\varepsilon \alpha)\left[-\left(u_{n}+K \sin v_{n}\right) \sin \alpha+v_{n} \cos \alpha\right]+\varepsilon v_{n} \sin \alpha,
\end{aligned}
$$

где отброшены слагаемые порядка малости $\varepsilon^{2}$ и выше, а также введены следующие безразмерные переменные:

$$
u=\frac{\dot{x}}{\omega_{0}}, \quad v=-x, \quad \alpha=\omega_{0} T, \quad \frac{\varepsilon}{2 \omega_{0}} \rightarrow \varepsilon
$$

Анализ системы (27) существенно опирается на тот факт, что ее якобиан тождественно равен $(1-\varepsilon \alpha)^{2}-\varepsilon^{2} \sin ^{2} \alpha$. Действительно, отсюда автоматически следует 
справедливость для данной системы аналога теоремы 3. Таким образом, интересующая нас проблема буферности сводится к поиску эллиптических состояний равновесия или циклов у отображения (27) при $\varepsilon=0$.

Как отмечено в [5], наибольший интерес представляют так называемые резонансные случаи $\alpha=2 \pi / q$, где $q$ - некоторое целое число, означающие, что период $T$ внешнего воздействия находится в отношении $1: q$ с периодом $2 \pi / \omega_{0}$ собственных колебаний осциллятора. В связи с этим рассмотрим, к примеру, случай $q=4$.

При $\varepsilon=0, \quad \alpha=\pi / 2$ система (27) переходит в отображение

$$
u_{n+1}=v_{n}, \quad v_{n+1}=-u_{n}-K \sin v_{n},
$$

которое, как нетрудно увидеть, при

$$
0<K<2, \quad K \neq \sqrt{2}
$$

имеет эллиптическую неподвижную точку $(u, v)=(0,0)$ и счетное число эллиптических циклов периода 4 с начальными условиями

$$
O_{(m, k)}=(m \pi, k \pi): \quad m, k \in \mathbb{Z}, \quad|m|+|k| \neq 0, \quad m+k \text { - четное. }
$$

Для получения более полного представления о динамике системы (28) обратимся к рис. 3, где показан фрагмент $\{(u, v): 0 \leqslant u \leqslant 14,0 \leqslant v \leqslant 14\}$ ее фазового портрета при $K=1.5$. Мы видим, что наблюдаются два принципиально различных типа движений: траектория либо совершает случайные блуждания по стохастической паутине, либо попадает в ловушку, т.е. находится в одном из островков устойчивости. Добавим еще, что самые крупные из таких ловушек примыкают к точкам (30) и к нулевому состоянию равновесия.

Перейдем теперь к отображению (27). Опираясь на аналог теоремы 3 , убеждаемся, что при условии $(29)$ и при $0<\varepsilon \ll 1,|\alpha-\pi / 2| \ll 1$ в нем реализуется феномен буферности: на месте ловушек образуются аттракторы, причем можно гарантировать существование любого наперед заданного конечного их числа. Отметим, что, как следует из содержащихся в [13] результатов численного анализа, буферность возникает в системе (27) и при других значениях $q$, например, в окрестности резонансов $1: q, 3 \leqslant q \leqslant 9$.

Подведем некоторый итог. Полученные в этом разделе результаты, несмотря на их математическую тривиальность, служат хорошей иллюстрацией механизма накапливания аттракторов в слабо диссипативных системах и, в частности, проливают свет на роль явления буферности в гамильтоновой динамике. В самом деле, при $\varepsilon=0$ отображения (3) и (27) переходят соответственно в отображение ЧириковаТейлора и отображение-паутину. Принято считать, что этим отображениям присущи типичные свойства физических систем. Кроме того, они служат базовыми моделями перехода в гамильтоновых системах с полутора степенями свободы от регулярных движений к стохастическим. Наши исследования подтверждают сделанный в [4], [5] вывод о том, что в реальных физических системах этот переход никогда не бывает полным, т.е. в море хаоса всегда существуют островки порядка. Действительно, в фазовом пространстве отображения (3) такие островки порядка 


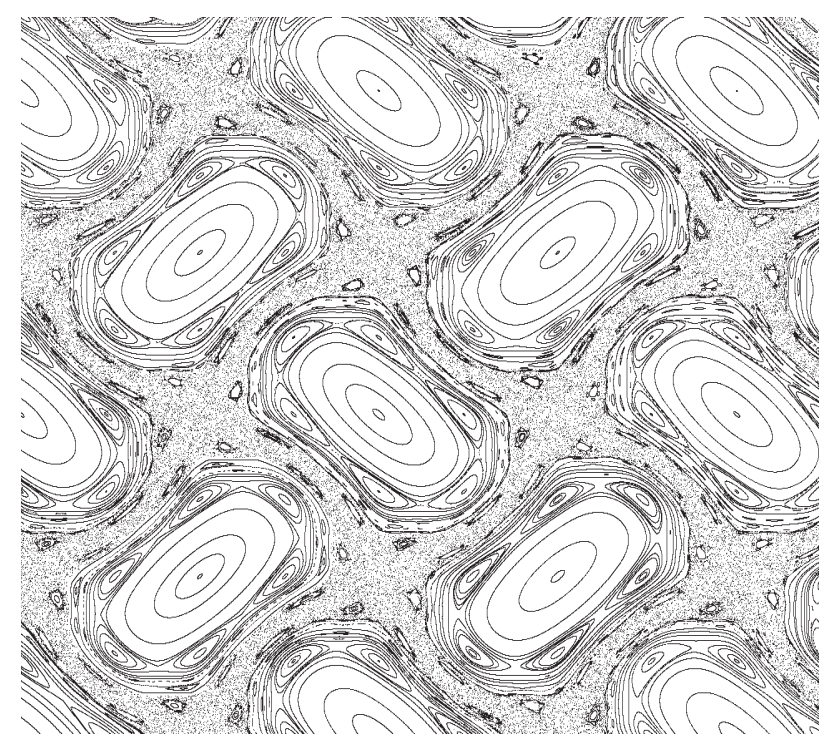

Рис. 3.

(устойчивые неподвижные точки) имеются при любом сколь угодно большом значении параметра стохастичности $K$ и, что самое интересное, при ослаблении диссипации их количество неограниченно растет. Впрочем, справедливости ради следует добавить, что при увеличении $K$ попасть на какой-либо из этих островков становится все сложнее. Например, как показал численный анализ, при $K=10, \varepsilon=0.0001$ основную область притяжения имеет хаотический аттрактор со старшим ляпуновским показателем $\lambda_{\max } \approx 1.62$.

\section{3. ФЕНОМЕН БУФЕРНОСТИ В $R L C$-АВТОГЕНЕРАТОРЕ С РЕЛЕЙНОЙ НЕЛИНЕЙНОСТЬЮ}

В этом разделе обнаруженный механизм возникновения буферности проиллюстрируем одной математической моделью из радиофизики. Рассмотрим автогенератор, блок-схема которого представлена на рис. 4а. Предположим, что вольтамперная характеристика его нелинейного элемента (в простейшем случае классической лампы) имеет вид, изображенный на рис. 4б. Пусть в цепь обратной связи этого генератора включен отрезок дискретной линии, состоящей из $N \geqslant 2$ одинаковых $R L C$-контуров (см. рис. 5). Тогда, опираясь на методику работы [14], можно показать, что при дополнительном предположении об идеальности усилителя математической моделью описанного генератора служит система

$$
\begin{aligned}
& \ddot{u}_{n}+\varepsilon \dot{u}_{n}+2 u_{n}-u_{n+1}-u_{n-1}=0, \quad n=1, \ldots, N, \\
& u_{N+1}=u_{N}, \quad u_{0}+\varphi\left(K u_{N}\right)=0 .
\end{aligned}
$$

Здесь $u_{n}$ - переменная нормированная составляющая напряжения в $n$-м контуре, $\varepsilon=R \sqrt{C / L}$ - активные потери в линии, которые всюду ниже мы предполагаем 


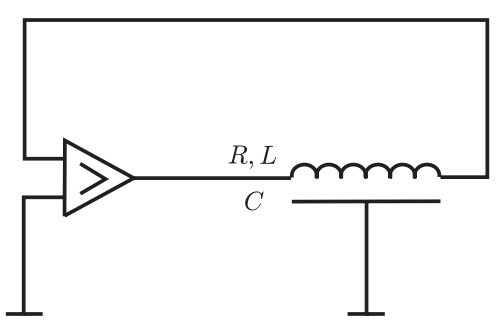

a)

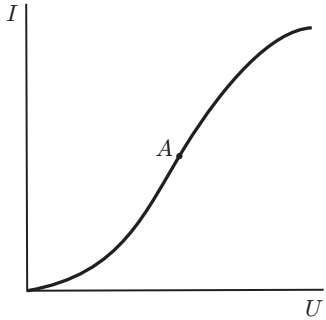

б)

Рис. 4.

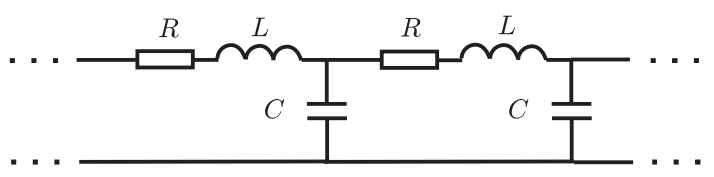

Рис. 5.

малыми, $K>0$ - коэффициент усиления, а функция $\varphi(z) \in C^{\infty}(\mathbb{R})$ получается из нелинейной характеристики усилителя в результате сдвига рабочей точки $A$ (см. рис. 4б) в начало координат. В связи с этим считаем, что $\varphi^{\prime}(z)>0$ при всех $z \in \mathbb{R}$, $\varphi(0)=0$ и, что самое главное, $\varphi(z) \rightarrow \pm 1$ при $z \rightarrow \pm \infty$.

Из перечисленных свойств нелинейности $\varphi(z)$ следует, что при $K \rightarrow \infty$ система (31) переходит в релейную систему

$$
\begin{aligned}
& \ddot{u}_{n}+\varepsilon \dot{u}_{n}+2 u_{n}-u_{n+1}-u_{n-1}=0, \quad n=1, \ldots, N, \\
& u_{N+1}=u_{N}, \quad u_{0}+\operatorname{sign}\left(u_{N}\right)=0,
\end{aligned}
$$

которая и является объектом дальнейшего анализа. Как правило, понятие решения такого типа уравнений проще всего определять конструктивно. Для того чтобы сделать это в нашем случае, сначала перепишем систему (32) в векторной форме

$$
\ddot{u}+\varepsilon \dot{u}+A u+a \cdot \operatorname{sign}(u, b)=0,
$$

где $u=\left(u_{1}, \ldots, u_{N}\right)^{\mathrm{T}}, \quad a=(1,0, \ldots, 0)^{\mathrm{T}}, \quad b=(0, \ldots, 0,1)^{\mathrm{T}}$,

$$
A=\left(\begin{array}{ccccccc}
2 & -1 & 0 & \ldots & \ldots & \ldots & 0 \\
-1 & 2 & -1 & 0 & \ldots & \ldots & 0 \\
0 & -1 & 2 & -1 & 0 & \ldots & 0 \\
\ldots & \ldots & \ldots & \ldots & \ldots & \ldots & \ldots \\
0 & \ldots & \ldots & 0 & -1 & 2 & -1 \\
0 & \ldots & \ldots & \ldots & 0 & -1 & 1
\end{array}\right) \text {, }
$$

$(\cdot, \cdot)$ - евклидово скалярное произведение в $\mathbb{R}^{N}$

Положим в $(33) \varepsilon=0$ и дополним получающуюся при этом консервативную систему

$$
\ddot{u}+A u+a \cdot \operatorname{sign}(u, b)=0
$$


произвольными начальными условиями

$$
\left.u\right|_{t=0}=u^{0}=\left(u_{1}^{0}, \ldots, u_{N}^{0}\right)^{\mathrm{T}},\left.\quad \dot{u}\right|_{t=0}=v^{0}=\left(v_{1}^{0}, \ldots, v_{N}^{0}\right)^{\mathrm{T}}
$$

из множества

$$
\Omega=\left\{\left(u^{0}, v^{0}\right) \in \mathbb{R}^{2 N}: u_{N}^{0}=0, \quad v_{N}^{0}>0\right\} .
$$

Тогда сначала, т.е. при не слишком больших положительных значениях $t$, решение задачи Коши (35), (36) совпадает с решением задачи Коши

$$
\ddot{u}+A u+a=0,\left.\quad u\right|_{t=0}=u^{0},\left.\quad \dot{u}\right|_{t=0}=v^{0} .
$$

Остановимся на некоторых необходимых для дальнейшего свойствах решения $u(t)$ задачи (38). Несложный анализ показывает, что матрица (34) является положительно определенной, а ее собственные значения $\lambda_{k}, k=1, \ldots, N$, имеют вид

$$
\lambda_{k}=2\left(1-\cos \theta_{k}\right), \quad \theta_{k}=\pi \frac{2 k-1}{2 N+1}, \quad k=1, \ldots, N .
$$

Поэтому для вектор-функции $u(t)$ справедливо равенство

$$
u(t)=-e+\cos (\sqrt{A} t)\left(u^{0}+e\right)+(\sqrt{A})^{-1} \sin (\sqrt{A} t) v^{0}
$$

где $e=(1, \ldots, 1)^{\mathrm{T}}, \sqrt{A}$ - арифметический квадратный корень из матрицы $A$. Для компоненты $u_{N}=u_{N}(t)$ этой вектор-функции из (40) вытекает представление вида

$$
u_{N}(t)=-1+\sum_{k=1}^{N} c_{k} \cos \omega_{k} t+d_{k} \sin \omega_{k} t
$$

где $\omega_{k}=\sqrt{\lambda_{k}}, \lambda_{k}$ определяются в формуле $(39)$.

В силу $(37) u_{N}(0)=0, \dot{u}_{N}(0)>0$. Поэтому заведомо существует интервал вида $\left(0, \delta_{0}\right), \quad \delta_{0}>0$, значений $t$, на котором $u_{N}(t)>0$. С другой стороны, функция $(41)$ представляет собой тригонометрический полином с отрицательным средним значением. Тем самым она должна иметь на полуоси $t>0$ нечетнократные нули. Пусть $t_{1}$ - наименьший из таких нулей, и его кратность равна $2 k_{0}-1$. Тогда для интересующего нас решения $u(t)$ релейной системы (35) формула (40) сохраняется на всем отрезке $0 \leqslant t \leqslant t_{1}$, а при значениях $t$, чуть больших $t_{1}$, это решение находится из задачи Коши

$$
\ddot{u}+A u-a=0,\left.\quad u\right|_{t=t_{1}}=u\left(t_{1}-0\right),\left.\quad \dot{u}\right|_{t=t_{1}}=\dot{u}\left(t_{1}-0\right) .
$$

Таким образом, при $t>t_{1},\left|t-t_{1}\right| \ll 1$ справедливо аналогичное (40) равенство

$$
u(t)=e+\cos \left[\sqrt{A}\left(t-t_{1}\right)\right]\left(u\left(t_{1}-0\right)-e\right)+(\sqrt{A})^{-1} \sin \left[\sqrt{A}\left(t-t_{1}\right)\right] \dot{u}\left(t-t_{1}\right) .
$$

Остановимся на естественно возникающем вопросе о разумности перехода от формулы (40) к формуле (43) при прохождении $t$ через значение $t_{1}$. С этой целью 
заметим, что поскольку функция (41) является решением линейного уравнения $\mathcal{L}\left(u_{N}\right)=0$, где

$$
\mathcal{L}=\frac{d}{d t} \cdot \prod_{k=1}^{N}\left(\frac{d^{2}}{d t^{2}}+\omega_{k}^{2}\right)
$$

то кратность любого ее нуля не превосходит $2 N$ и, в частности, $k_{0} \leqslant N$. Кроме того, две различные формулы для $u_{N}(t)$, получающиеся соответственно из (40) и $(43)$, в точке $t=t_{1}$ "склеиваются" $C^{2 N-1}$-гладким образом, т.е.

$$
u_{N}^{(k)}\left(t_{1}-0\right)=u_{N}^{(k)}\left(t_{1}+0\right), \quad k=0,1, \ldots, 2 N-1 .
$$

Для доказательства этих соотношений заметим, что фигурирующие в (38) и (42) системы отличаются только уравнениями для компоненты $u_{1}$. Поэтому как при $t<t_{1}$, так и при $t>t_{1}$ имеют место одни и те же равенства

$$
u_{N}^{(2 k)}(t)=\Delta^{k} u_{N}(t), \quad u_{N}^{(2 k+1)}(t)=\Delta^{k} \dot{u}_{N}(t), \quad k=1, \ldots, N-1,
$$

где $\Delta-$ разностный оператор вида

$$
\Delta u_{j}=u_{j+1}-2 u_{j}+u_{j-1}, \quad j=1, \ldots, N, \quad u_{N+1}=u_{N}, \quad u_{0}=0 .
$$

А отсюда и из начальных условий в (42) требуемые свойства (44) получаются уже очевидным образом.

Из проделанных построений вытекает, что

$$
u_{N}(t)=c_{k_{0}}\left(t-t_{1}\right)^{2 k_{0}-1}+o\left(\left(t-t_{1}\right)^{2 k_{0}-1}\right), \quad t \rightarrow t_{1}+0,
$$

где в силу (44) и способа выбора $t_{1}$ имеем

$$
c_{k_{0}}=\frac{u_{N}^{\left(2 k_{0}-1\right)}\left(t_{1}+0\right)}{\left(2 k_{0}-1\right) !}=\frac{u_{N}^{\left(2 k_{0}-1\right)}\left(t_{1}-0\right)}{\left(2 k_{0}-1\right) !}<0 .
$$

Таким образом, заведомо существует интервал вида $\left(t_{1}, t_{1}+\delta_{0}\right), \delta_{0}>0$, на котором $u_{N}(t)<0$, и, следовательно, переход от задачи (38) к (42) оказывается законным. В дальнейшем такого рода переходы будем называть переключениями. Кроме того, переключение, происходящее в некоторой точке $t_{m}$, назовем нормальным, если на интервале между соседними переключениями $t_{m-1}$ и $t_{m}, t_{m-1}<t_{m}$, функция $u_{N}(t)$ не имеет четнократных нулей, а в самой точке $t_{m}$ выполняется неравенство $\dot{u}_{N}\left(t_{m}\right) \neq 0$.

Последующие построения полностью аналогичны уже изложенным. Действительно, при $t>t_{1}$ для компоненты $u_{N}(t)$ вектор-функции (43) справедливо представление

$$
u_{N}(t)=1+\sum_{k=1}^{N} \tilde{c}_{k} \cos \omega_{k} t+\tilde{d}_{k} \sin \omega_{k} t,
$$

где частоты $\omega_{k}$ те же, что и в (41). Далее заметим, что поскольку $u_{N}(t)<0$ при значениях $t$, близких к $t_{1}$, а среднее значение тригонометрического полинома (45) 
положительно, то на полуоси $\left(t_{1},+\infty\right)$ у функции $u_{N}(t)$ с необходимостью существуют нечетнократные нули. Обозначим через $t_{2}$ наименьший из этих нулей. Рассуждая, как и выше, убеждаемся, что формула (43) справедлива только на отрезке $t_{1} \leqslant t \leqslant t_{2}$, а при $t=t_{2}$ происходит очередное переключение. Таким образом, при $t>t_{2}$ (до следующего переключения) решение $u(t)$ задачи (35), (36) определяется из задачи Коши

$$
\ddot{u}+A u+a=0,\left.\quad u\right|_{t=t_{2}}=u\left(t_{2}-0\right),\left.\quad \dot{u}\right|_{t=t_{2}}=\dot{u}\left(t_{2}-0\right)
$$

и т.д.

На основании проделанного анализа заключаем, что решение $u(t)$ релейной системы (35) с произвольными начальными условиями из множества (37) существует и единственно при всех $t \geqslant 0$ и имеет бесконечную последовательность переключений $t_{m}, m \geqslant 1$. Поэтому, в частности, корректно определен $(2 N-1)$-мерный оператор

$$
S:\left(u^{0}, v^{0}\right) \in \Omega \rightarrow\left(u\left(t_{2}\right), \dot{u}\left(t_{2}\right)\right) \in \bar{\Omega}
$$

первого возвращения в $\bar{\Omega}$ по траекториям системы (35).

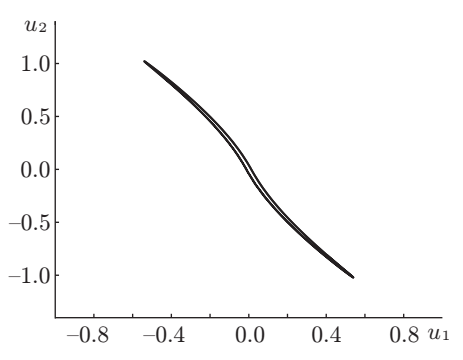

а) Два переключения

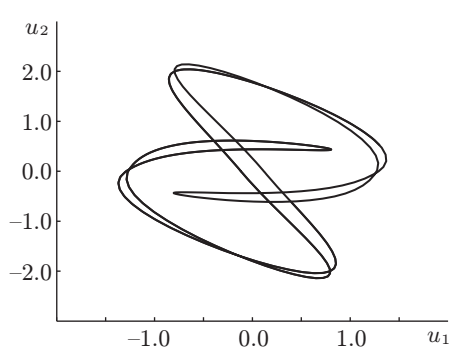

в) Четыре переключения

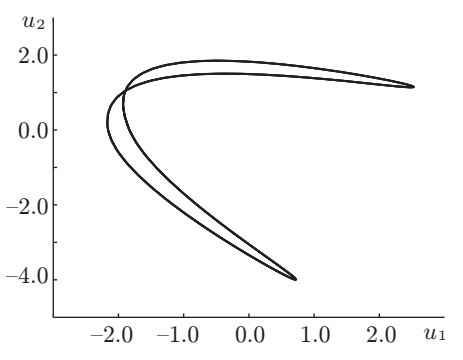

б) Два переключения

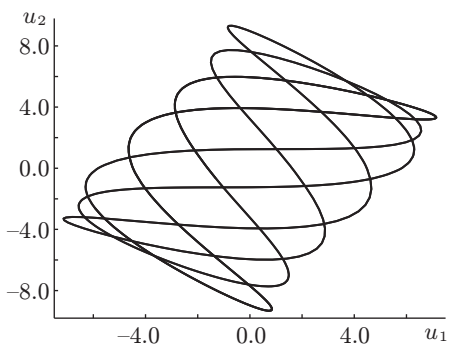

г) Шесть переключений

Рис. 6. Случай $N=2, \varepsilon=0.01$.

Остановимся на некоторых свойствах оператора (46). Отметим, во-первых, что он бесконечно дифференцируем в окрестности любой точки $\left(u^{0}, v^{0}\right)$, для которой переключения при $t=t_{1}$ и $t=t_{2}$ являются нормальными. В точках $\left(u^{0}, v^{0}\right)$, где нарушается нормальность хотя бы одного из упомянутых переключений, отображение (46), вообще говоря, оказывается разрывным. Во-вторых, оператор $S$ корректно 
определен и в случае $\varepsilon>0$, в чем нетрудно убедиться, повторяя практически дословно все проведенные выше построения. И наконец, при $\varepsilon=0$ он сохраняет фазовый объем (в силу консервативности системы (35)), а при малых $\varepsilon>0$ становится слабо диссипативным.

Итак, $(2 N-1)$-мерное отображение $(46)$ обладает при $\varepsilon=0$ и $\varepsilon>0$ теми же общими свойствами, что и отображения $(3),(21),(27)$. Поэтому есть все основания ожидать, что, как и в упомянутых двумерных системах, при $\varepsilon \rightarrow 0$ количество его аттракторов неограниченно растет. Следует, однако, заметить, что строгое рассмотрение данного вопроса сопряжено со значительными аналитическими трудностями даже в простейшем случае $N=2$. В связи с этим ограничимся изложением результатов численного интегрирования системы (32) при $N=2$ и $N=12$.

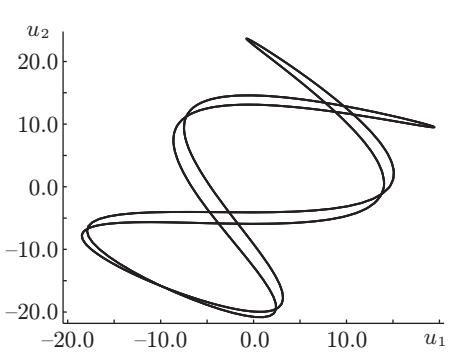

а) Четыре переключения

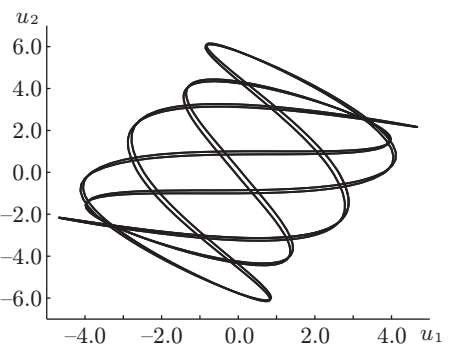

в) Десять переключений

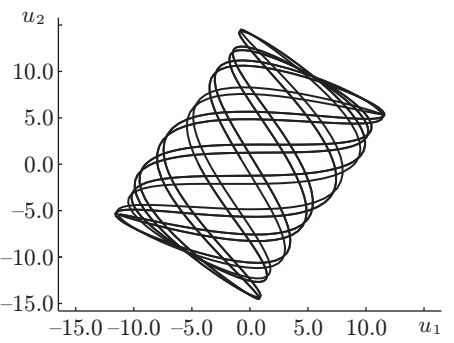

д) Четырнадцать переключений

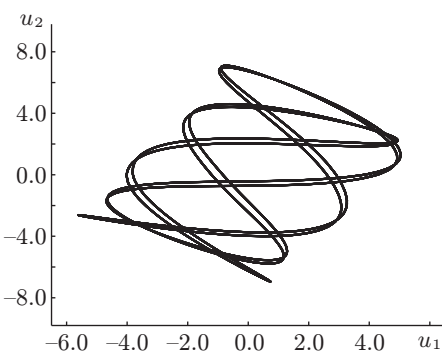

б) Восемь переключений

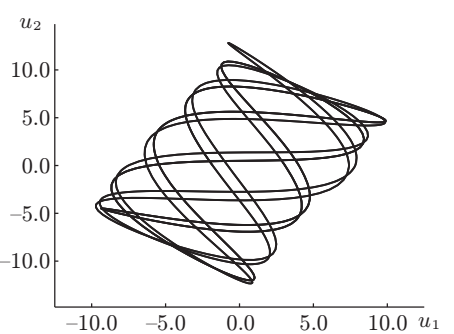

г) Десять переключений

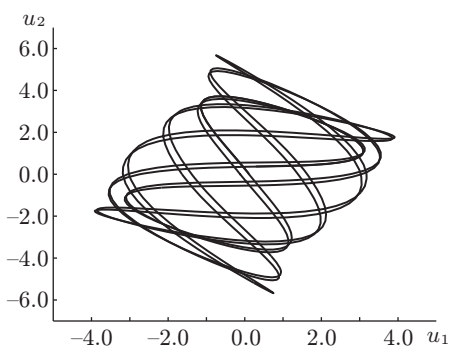

е) Четырнадцать переключений

Рис. 7. Случай $N=2, \varepsilon=0.001$. 
Численный анализ, проделанный с использованием пакетов программ tracer 3.47 (tracer3.narod.ru) и matds (www.math.rsu.ru/mexmat/kvm/matds), показал, что при $N=2, \varepsilon=0.1$ система (32) имеет, по всей видимости, единственный устойчивый цикл, но уже при $N=2, \varepsilon=0.01$ ситуация принципиально меняется. В этом случае удается обнаружить не менее пяти устойчивых циклов разной степени сложности. Проекции четырех из них на плоскость $\left(u_{1}, u_{2}\right)$ представлены на рис. 6.

Следует отметить, что некоторые из найденных циклов (рис. 6а, в, г) являются самосимметричными, т.е. не меняются при замене

$$
u_{1} \rightarrow-u_{1}, \quad u_{2} \rightarrow-u_{2}
$$

Цикл, показанный на рис. 6б, существует в паре с симметричным ему устойчивым циклом, получающимся в результате преобразования (47). Будем далее характеризовать сложность того или иного цикла числом его переключений на отрезке длины периода, которое, заметим, всегда четно. Например, если упомянутое число равно $2 m_{0}$, то это означает, что такому циклу соответствует неподвижная точка оператора $S^{m_{0}}$, где, напомним, $S$ - отображение первого возвращения (46). При $N=2$, $\varepsilon=0.01$ самым сложным из всех устойчивых режимов, которые удалось выявить, оказался цикл с шестью переключениями (рис. 6г).

Дальнейшее уменьшение $\varepsilon$ в полном соответствии с выработанными в разделе 2 теоретическими представлениями приводит к увеличению числа аттракторов. При $N=2, \quad \varepsilon=0.001$, к примеру, помимо уже найденных ранее пяти появляются еще не менее девяти устойчивых циклов. Проекции шести из них изображены на рис. 7 (как и в предыдущем случае, опущены парные циклы, имеющиеся у циклов, показанных на рис. 7а, б, г). Добавим, что при уменьшении $\varepsilon$ происходит не только неограниченное возрастание общего числа аттракторов, но и возникают все более сложно устроенные периодические режимы. В частности, при $N=2, \varepsilon=0.0001$ имеются устойчивые циклы с числом переключений, равным 16, 18, 22, 26, 34, 38 .

Приведенные результаты численного расчета свидетельствуют о том, что общий механизм накапливания устойчивых циклов, характерный для произвольных слабо диссипативных систем, реализуется в системе (32) уже в простейшем случае $N=$ 2. Однако в данной задаче существует и альтернативный механизм возникновения феномена буферности, наблюдающийся при увеличении числа $N$ звеньев в линии и при фиксированном $\varepsilon>0$. Действительно, при $N=12, \varepsilon=0.01$ система (32) имеет не менее 11 аттракторов (10 циклов и один двумерный тор), т.е. при увеличении $N$ мы добиваемся того же эффекта, что и в случае $N=2$ при последовательном уменьшении $\varepsilon$. Проекции некоторых из этих аттракторов показаны на рис. 8 .

Причины, по которым происходит увеличение числа аттракторов при $N \rightarrow \infty$, становятся понятными, если рассмотреть отвечающую системе (31) распределенную модель

$$
u_{t t}+\varepsilon u_{t}=u_{x x},\left.\quad u_{x}\right|_{x=1}=0,\left.\quad u\right|_{x=0}+\varphi\left(\left.K u\right|_{x=1}\right)=0,
$$

где дискретная линия заменена отрезком $0 \leqslant x \leqslant 1$ длинной линии с равномерно распределенными электрическими параметрами $L, C, R$, а нелинейная характеристика $\varphi(z)$ помимо перечисленных выше свойств удовлетворяет еще условию $\varphi^{\prime \prime \prime}(0)<0$. Тогда, как установлено в [2], при увеличении коэффициента усиления $K$ 


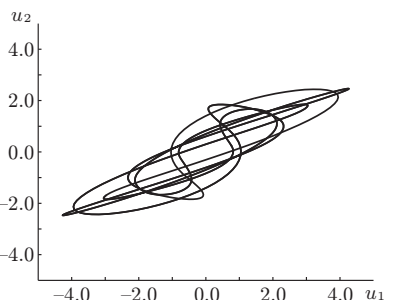

а) Десять переключений

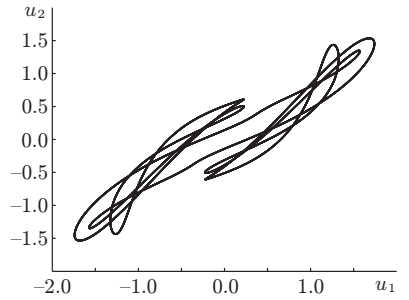

в) Два переключения

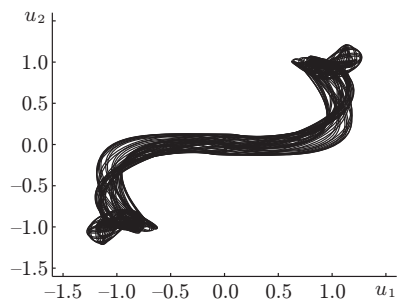

д) Двумерный тор

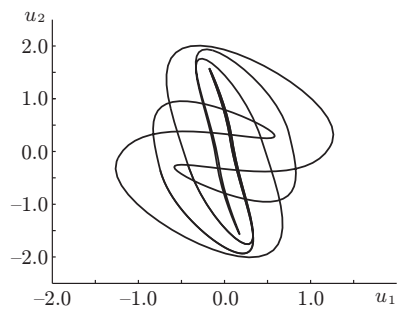

ж) Десять переключений

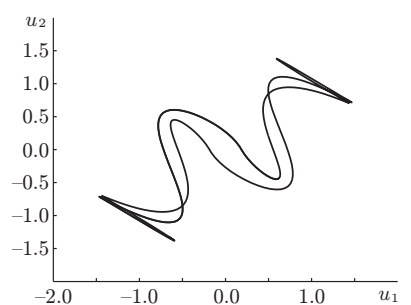

и) Два переключения

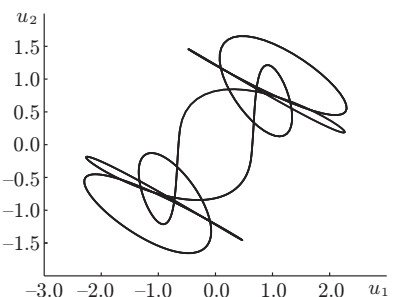

б) Два переключения

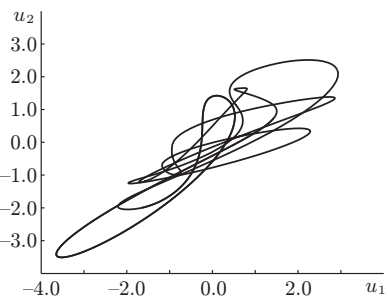

г) Восемь переключений

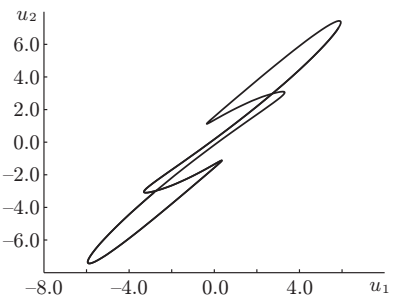

е) Два переключения

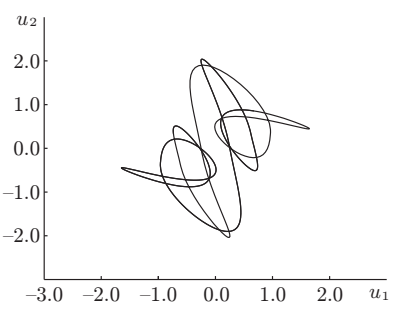

з) Шесть переключений

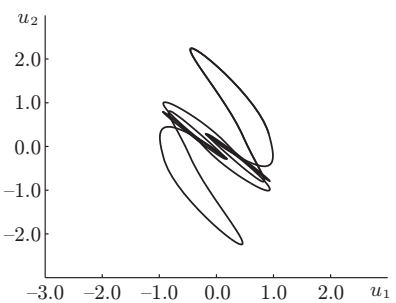

к) Шесть переключений

Рис. 8. Случай $N=12, \varepsilon=0.01$. 
и при прохождении его через некоторые критические значения от нулевого состояния равновесия краевой задачи (48) последовательно ответвляются неустойчивые циклы, причем каждый отдельно взятый из них обретает устойчивость, вырастая по амплитуде. Что касается дискретной модели (31), то при больших $N$ она имеет аналогичную динамику по параметру $K$.

\section{4. ЗАКЛЮЧЕНИЕ}

Накопленный к настоящему времени достаточно богатый фактический материал, частично представленный в работах [1]-[3], позволяет утверждать, что механизм возникновения буферности, обнаруженный в краевой задаче (48) при увеличении $K$, характерен для широкого класса радиофизических систем, математическими моделями которых служат линейные телеграфные уравнения с нелинейными граничными условиями на концах конечного отрезка. Однако, как показывают проделанные выше исследования, этот механизм не является единственно возможным. По всей видимости, в реальных радиофизических системах квазигармонические циклы, испытывающие бифуркацию в нуле и обретающие впоследствии устойчивость, сосуществуют с нелокальными аттракторами, происхождение которых связано с близостью системы к консервативной. Во всяком случае, именно такая ситуация реализуется в рамках дискретной модели (31) при достаточно больших $N$, малых $\varepsilon>0$ и при различных значениях $K>0$ (в том числе и при $K<1 / \varphi^{\prime}(0)$, когда устойчиво нулевое решение). Например, при $N=12, \varepsilon=0.01, K=1.8, \varphi(z)=$ th $z$ в системе (31) сосуществуют не менее девяти аттракторов: пять циклов, три двумерных тора и один трехмерный тор. В случае $N=12, \varepsilon=0.001, \quad K=0.2, \quad \varphi(z)=$ th $z$ помимо устойчивого нулевого состояния равновесия удалось обнаружить еще четыре аттрактора: три двумерных тора и один трехмерный тор (в каждом случае вычислялись ляпуновские показатели и ляпуновская размерность).

В заключение приведем ряд дополнительных аргументов, свидетельствующих о наличии у краевой задачи (48) устойчивых докритических режимов (т.е. аттракторов, сосуществующих с устойчивым нулевым состоянием равновесия). Для этого проинтегрируем фигурирующее в (48) линейное уравнение методом Римана. В результате убеждаемся, что любое решение указанного уравнения, удовлетворяющее граничному условию $\left.u_{x}\right|_{x=1}=0$, с точностью до членов порядка $\varepsilon^{2}$ имеет вид

$$
u=e^{-\varepsilon t / 2}[h(t+(x-1))+h(t-(x-1))],
$$

где $h(t)$ - произвольная непрерывная функция (здесь речь идет об обобщенных решениях из класса $C$ ). Подставляя формулу (49) во второе граничное условие из (48) и полагая

$$
\theta(t)=e^{-\varepsilon t / 2} h(t), \quad \eta(t)=\theta(t-1),
$$

приходим к системе разностных уравнений

$$
\eta(t+1)=\theta(t), \quad \theta(t+1)=-e^{-\varepsilon} \eta(t)-e^{-\varepsilon / 2} \varphi(2 K \theta(t)) .
$$

Теория разностных уравнений с непрерывным временем развита в книге [15]. Напомним, что произвольной паре непрерывных при $-1 \leqslant t \leqslant 0$ начальных функций 
$\eta_{0}(t), \theta_{0}(t)$, удовлетворяющих условиям согласования

$$
\eta_{0}(0)=\theta_{0}(-1), \quad \theta_{0}(0)=-e^{-\varepsilon} \eta_{0}(-1)-e^{-\varepsilon / 2} \varphi\left(2 K \theta_{0}(-1)\right),
$$

соответствует единственная пара непрерывных при всех $t \geqslant 0$ функций $\eta(t), \theta(t)$ таких, что $\eta(0)=\eta_{0}(0), \theta(0)=\theta_{0}(0)$, обращающих уравнения $(50)$ в тождества. Напомним также, что поскольку эти функции строятся методом шагов, то их поведение при $t \rightarrow \infty$ определяется поведением итераций соответствующего дискретного отображения

$$
\eta_{n+1}=\theta_{n}, \quad \theta_{n+1}=-e^{-\varepsilon} \eta_{n}-e^{-\varepsilon / 2} \varphi\left(2 K \theta_{n}\right),
$$

которое после нормировок и переобозначений

$$
\eta e^{\varepsilon / 2} \rightarrow \eta, \quad \theta e^{\varepsilon / 2} \rightarrow \theta, \quad 2 K e^{-\varepsilon / 2}=\beta, \quad \mu=1-e^{-\varepsilon} \ll 1
$$

преобразуется к виду

$$
\eta_{n+1}=\theta_{n}, \quad \theta_{n+1}=-(1-\mu) \eta_{n}-\varphi\left(\beta \theta_{n}\right) .
$$

Получившееся отображение при $\mu=0$ сохраняет площадь, а при $\mu>0$ становится слабо диссипативным, так как его якобиан тождественно равен $1-\mu$. Таким образом, для данного отображения остается в силе аналог теоремы 3, и вследствие этого проблема буферности для него сводится к отысканию эллиптических циклов при $\mu=0$.

Характер поведения траекторий отображения (51) в докритическом случае, т.е. при $\beta<2 / \varphi^{\prime}(0)$, проясняет рис. 9, на котором изображен фрагмент $\{(\eta, \theta):|\eta| \leqslant 2$, $|\theta| \leqslant 2\}$ его фазового портрета при $\mu=0, \varphi(z)=$ th $z, \beta=1.5$. В самом деле, при малых $\mu$ нулевая неподвижная точка становится устойчивым фокусом, но кроме нее появляются еще и устойчивые циклы, возникающие на месте соответствующих островков устойчивости (эллиптических циклов). Некоторые из этих островков показаны на рис. 9 .

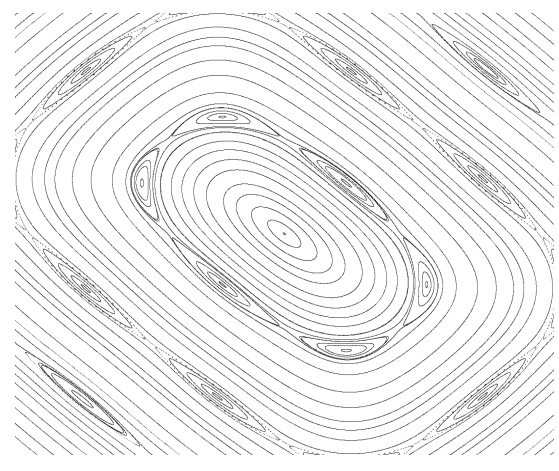

Рис. 9

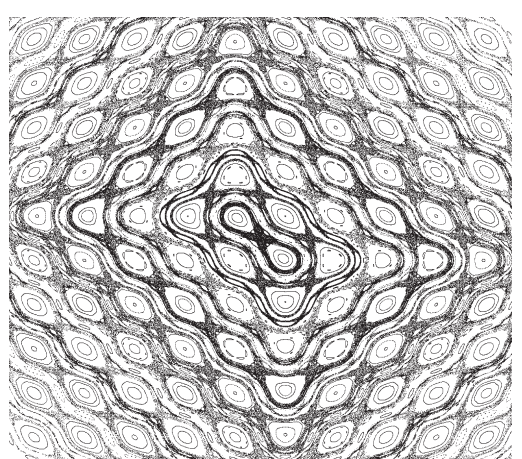

Рис. 10

При переходе в закритическую область значений параметра $\beta$ феномен буферности в отображении (51) проявляется более ярко, т.е. заведомо увеличивается количество устойчивых циклов, "выживающих" при малых $\mu>0$. Для того чтобы 
убедиться в этом, достаточно обратиться к рис. 10, где показан фрагмент $\{(\eta, \theta)$ : $|\eta| \leqslant 5,|\theta| \leqslant 5\}$ фазового портрета системы (51) в случае $\mu=0, \quad \varphi(z)=$ th $z$, $\beta=3.5$. Заметим, что здесь из-за наличия стохастического слоя число островков устойчивости при $\mu=0$, вообще говоря, счетно.

Благодарности. Авторы выражают глубокую признательность рецензенту за внимательное ознакомление с рукописью и высказанные конструктивные замечания, способствовавшие улучшению изложения. Работа выполнена при финансовой поддержке РФФИ (грант № 05-01-01004).

\section{Список литературы}

[1] A. A. Bumm. ЖТТ. 1934. Т. 4. № 1. С. 144-157.

[2] А. Ю. Колесов, Н. Х. Розов. Труды МИАН. 2001. Т. 233. С. 153-207.

[3] А. Ю. Колесов, Е. Ф. Мищенко, Н. Х. Розов. Труды МИАН. 1998. Т. 222. С. 1-193; А. Ю. Колесов, Н. Х. Розов, В. Г. Сушко. Фунд. и прикл. математика. 1999. Т. 5. № 2. С. 437-473; А. Ю. Колесов, Е. Ф. Мищенко, Н. Х. Розов. УМН. 2000. Т. 55. № 2(332). С. 95-120; А. Ю. Колесов, Н. Х. Розов. ПММ. 2001. Т. 65. № 2. C. $183-198$.

[4] Г. М. Заславский, Р. З. Сагдеев. Введение в нелинейную физику. От маятника до турбулентности и хаоса. М.: Наука, 1988.

[5] Г. М. Заславский. Физика хаоса в гамильтоновых системах. Москва-Ижевск: Ин-т компьютерных исследований, 2004.

[6] Н. К. Гаврилов, Л. П. Шильников. Матем. сб. 1972. Т. 88. № 4. С. 475-492; 1973. T. 90. № 1. C. 139-157.

[7] И. М. Овсянников, Л. П. Шильников. Матем. сб. 1986. Т. 130. № 4. С. 552-570.

[8] И. М. Овслнников, Л. П. Шильников. Матем. сб. 1991. Т. 182. № 7. С. 1043-1073.

[9] S. E. Newhouse. Publ. Math. IHES. 1979. V. 50. P. 101-151; Lectures on dynamical systems. In: Dynamical Systems. C.I.M.E. Lectures (Bressanone, Italy, June, 1978). Progr. Math. V. 8. Eds. J. Guckenheimer, J. Moser, S. E. Newhouse. Boston: Birkhäuser, 1980. P. 1-114.

[10] Дж. Гукенхеймер, Ф. Холмс. Нелинейные колебания, динамические системы и бифуркации векторных полей. Москва-Ижевск: Ин-т компьютерных исследований, 2002.

[11] А. Ю. Колесов, Н. Х. Розов. Инвариантные торы нелинейных волновых уравнений. М.: Физматлит, 2004; Е. Ф. Мищенко, В. А. Садовничий, А. Ю. Колесов, Н. Х. Розов. Автоволновые процессы в нелинейных средах с диффузией. М.: Физматлит, 2005.

[12] А. Лихтенберг, М. Либерман. Регулярная и стохастическая динамика. Череповец: Меркурий-Пресс, 2000.

[13] А. Д. Морозов, Т. Н. Драгунов. Визуализация и анализ инвариантных множеств динамических систем. Москва-Ижевск: Ин-т компьютерных исследований, 2003.

[14] Ю. С. Колесов. Математическая теория $R C$-автогенераторов с распределенными параметрами в цепи обратной связи. В сб.: Дифференциальные уравнения и их применение. Вып. 2. Ред. М. Сапаговас. Вильнюс: Ин-т физики и математики АН Лит. ССР, 1971. C. $1-67$.

[15] А. Н. Шарковский, Ю. Л. Майстренко, Е. Ю. Романенко. Разностные уравнения и их приложения. Киев: Наукова думка, 1986.

Поступила в редакцию 11.II.2005 г., после доработки 16.VI.2005 г. 